\title{
Global haemostatic tests demonstrate the absence of parameters of hypercoagulability in non-hypoxic mild COVID-19 patients: a prospective matched study
}

\author{
Syed Nabeel Muzaffar ${ }^{1}\left[\right.$. Suhail Sarwar Siddiqui ${ }^{1} \cdot$ Afzal Azim $^{2}$
}

Accepted: 14 November 2021 / Published online: 20 November 2021

(c) The Author(s), under exclusive licence to Springer Science+Business Media, LLC, part of Springer Nature 2021

\section{Dear Editor,}

We read with great interest the article published by Fan et al. in your esteemed journal in the September 2021 issue, entitled "Global haemostatic tests demonstrate the absence of parameters of hypercoagulability in non-hypoxic mild COVID-19 patients: a prospective matched study" [1]. In this study, authors have done a commendable job of identifying and classifying coagulopathy in COVID-19 patients by using thromboelastography (TEG). TEG is a comprehensive test which analyzes whole coagulation cascade and deciphers abnormalities pertaining to specific components of coagulation system unlike conventional coagulation assays (CCAs), which depict only the initial phase of clotting cascade and are less informative [2]. Microthrombosis secondary to hypercoagulation is an essential feature of COVID-19 associated coagulopathy [3] and TEG may play a pivotal role in identification of hypercoagulability [4]. In this study, the authors analyzed patients with mild and severe illnesses admitted to intensive care unit (ICU) and found a hypercoagulable thromboelastography (TEG) profile in severe COVID19 patients. However, there are a few points which we would like to emphasize upon which are as mentioned below:

1. Study population:

Syed Nabeel Muzaffar

muzaffarnabeel@gmail.com

Suhail Sarwar Siddiqui

sarwarsuhail@gmail.com

Afzal Azim

draazim2002@gmail.com

1 Department of Critical Care Medicine, King George's Medical University, Lucknow, Uttar Pradesh 226003, India

2 Department of Critical Care Medicine, Sanjay Gandhi Postgraduate Institute of Medical Sciences (SGPGIMS), Lucknow, Uttar Pradesh 226014, India (a) As the study has been done in Asian population, it would be difficult to extrapolate the study findings on a generalized basis.

(b) The patients (in severe category) for whom prophylactic anticoagulation had already been started were not excluded from the study. Thus, extrapolation of study findings to all COVID-19, especially those not receiving any anticoagulation, is difficult.

2. Baseline TEG values:

The normal baseline values for TEG in healthy controls have not yet been validated and may vary between different populations. Studies are required to establish the baseline values and then decide test cut-offs accordingly [5].

3. Methodology:

(a) Sample collection technique with respect to the amount of blood collected in syringe, type of vial used for storing blood, transportation time to TEG lab and type of TEG machine used (heparinase or kaolin-based) have an important influence on study results. This needs a detailed explanation in methodology section [2].

(b) As we know, adequate (as per recommended guidelines) handling, storage, transportation and disposal of COVID-19 positive patient's blood sample and disinfection of TEG machine are very essential as blood is a source of infection for both the health care staff and environment. This needs to be described meticulously in the methodology [6].

4. Cross-over:

During the course of illness, whether any patient in mild COVID-19 category worsened and crossed over 
to the severe COVID-19 category is not mentioned. Such a cross-over can have implications on interpretation of study results.

5. Co-existing infections as confounding factors (as there is an intricate interplay between inflammation and coagulation) [2]:

As we have seen in COVID-19 pandemic, concomitant multidrug resistant bacterial infections and fungal infections may emerge during the course of illness. Whether patients in this study had such coexisting infections or not could have further helped us in attributing derangements in TEG parameters to underlying COVID-19 infection per se and not to other confounding factors.

6. Dynamics of coagulation:

It is worth appreciating that TEG parameters have been sequentially examined which enlightens the dynamics of coagulation during disease course. However, serial TEG has been done for severe COVID-19 patients only and not for patients in the mild category, which could have further compared the spectrum and trend of coagulopathy between these groups.

7. Hypocoagulability:

In this study, hypercoagulability was seen in severe category, which can guide us in titrating the anticoagulation therapy to the underlying TEG pattern. However, not only hypercoagulability, but also hypocoagulability can be seen in severe COVID-19 patients in conditions like disseminated intravascular coagulopathy (DIC) [3].

Hypocoagulable TEG pattern has been associated with poor outcome in patients with sepsis and may be useful in risk-stratification of COVID-19 patients also [7, 8]. In addition, blood component therapy can be tailored to the specific clotting pathway affected (clot formation, clot kinetics or clot strength), which depends upon clotting factors, fibrinogen and platelets respectively.

8. Coagulation index (CI) as a single TEG metric:

Rather than analyzing individual values, $\mathrm{CI}$ combines several TEG parameters and presents the complete TEG picture in a single value. Therefore, just a single CI value can quickly classify TEG patterns into normo-coagulable, hypo-coagulable or hyper-coagulable states [2].

9. Clot dissolution:

It is worth appreciating that Clot lysis index (LY30) to assesses fibrinolysis has also been done in this study, which informs us about breakdown of clot after its formation and assesses the clot stability. Hyperfibrinolysis has been noticed in conditions like cirrhosis and trauma. Adamzik et al. compared TEG parameters between post-operative patients and those with sep- sis and found lysis index to be significantly increased (hyperfibrinolysis) in patients with sepsis suggesting an early involvement of fibrinolytic pathway in sepsis [7]. Even in patients with COVID-19, it has been seen that found that there is an involvement of fibrinolytic pathway [9]. Using indices like LY30 in these patients (which can be analyzed by TEG unlike CCA), can help us understand the spectrum of changes in fibrinolysis also (hyperfibrinolysis or hypofibrinolysis).

10. Platelet function:

TEG is not a good test for assessing platelet function for which tests like platelet aggregometry are preferred [10].

11. Prognostication of outcome:

Correlation of TEG with sequential Organ Failure Assessment (SOFA) score at admission and during disease course along with comparison of TEG parameters between survivors and non-survivors could have given further useful information about the association between dynamics of coagulopathy and disease outcome.

A similar study was published by us in 2017 in which we assessed spectrum of sepsis-induced coagulopathy in nonbleeding adult septic patients, where TEG was compared with CCAs at ICU admission [11]. It was found that even patients with normal coagulation assays (on the basis of CCAs) could be further categorized into different profiles based on TEG values like normocoagulable, hypocoagulable and hypercoagulable states. Moreover, patients with hypocoagulability were also found to have mixed abnormalities in the form of an underlying hypercoagulable or normocoagulable state. Hypocoagulable TEG pattern was seen in patients with septic shock which corroborates with the finding of similar other studies $[7,8]$. TEG is popular in the fields of cardiac surgery and trauma and would also play a useful role in the management of COVID-19 associated coagulopathy, for which further large scale research is required.

Author contributions Material preparation, data collection and analysis were performed by SNM and SSS. The first draft of the manuscript was written by SNM and all authors commented on previous versions of the manuscript. All authors read and approved the final manuscript.

\section{Declarations}

Conflict of interest The authors declare no conflict of interest.

\section{References}

1. Fan BE, Ramanathan K, Sum CLL et al (2021) Global haemostatic tests demonstrate the absence of parameters of hypercoagulability 
in non-hypoxic mild COVID-19 patients: a prospective matched study. J Thromb Thrombolysis. https://doi.org/10.1007/ s11239-021-02575-4

2. Afzal A, Syed NM (2017) Thromboelastography and thromboelastometry in patients with sepsis-a mini-review. J Anesth Intensive Care Med 3:555603. https://doi.org/10.19080/JAICM.2017. 03.555603

3. Leentjens J, van Haaps TF, Wessels PF, Schutgens REG, Middeldorp S (2021) COVID-19-associated coagulopathy and antithrombotic agents-lessons after 1 year. Lancet Haematol 8:e524-e533. https://doi.org/10.1016/S2352-3026(21)00105-8

4. Panigada M, Bottino N, Tagliabue P, Grasselli G, Novembrino C, Chantarangkul V, Pesenti A, Peyvandi F, Tripodi A (2020) Hypercoagulability of COVID-19 patients in intensive care unit: a report of thromboelastography findings and other parameters of hemostasis. J Thromb Haemost 18:1738-1742. https://doi.org/10. $1111 /$ jth. 14850

5. Muzaffar SN, Azim A, Siddiqui SS (2021) Re: thromboelastograph: a prognostic marker in sepsis with organ dysfunction without overt bleeding. J Crit Care. https://doi.org/10.1016/j.jcrc.2021. 09.003

6. (2020) Infection prevention and control (IPC) for novel coronavirus (COVID-19). JAC Antimicrob Resist 2:dlaa027. https://doi. org/10.1093/jacamr/dlaa027

7. Adamzik M, Eggmann M, Frey UH, Görlinger K, Bröcker-Preuss M, Marggraf G et al (2011) Comparison of thromboelastometry with simplified acute physiology score II and sequential organ failure assessment scores for the prediction of 30-day survival: a cohort study. Shock 4:339-342. https://doi.org/10.1097/SHK. 0b013e318204bff6

8. Ninan KF, Iyadurai R, Varghese JK, Geevar T, Nair SC, Yadav B et al (2021) Thromboelastograph: a prognostic marker in sepsis with organ dysfunction without overt bleeding. J Crit Care 65:177-183. https://doi.org/10.1016/j.jcrc.2021.06.005

9. Coccheri S (2020) COVID-19: the crucial role of blood coagulation and fibrinolysis. Intern Emerg Med 15:1369-1373. https:// doi.org/10.1007/s11739-020-02443-8

10. Paniccia R, Priora R, Liotta AA, Abbate R (2015) Platelet function tests: a comparative review. Vasc Health Risk Manag 11:133-148. https://doi.org/10.2147/VHRM.S44469

11. Muzaffar SN, Baronia AK, Azim A, Verma A, Gurjar M, Poddar B et al (2017) Thromboelastography for evaluation of coagulopathy in nonbleeding patients with sepsis at Intensive Care Unit admission. Indian J Crit Care Med 21:268-273. https://doi.org/10. 4103/ijccm.IJCCM_72_17

Publisher's Note Springer Nature remains neutral with regard to jurisdictional claims in published maps and institutional affiliations. 